\title{
Surface Dynamics of the Cyclohexadienyl Radical Adsorbed on Silica Gel Investigated Using Avoided Level-Crossing Muon Spin Resonance
}

\author{
By Martina Schwager, Emil Roduner*
}

Physikalisch-Chemisches Institut der Universität Zürich, Winterthurerstrasse 190, CH-8057 Zürich, Switzerland

Ivan D. Reid

Paul Scherrer Institute, CH-5232 Villigen PSI, Switzerland

\author{
Sydney R. Kreitzman
}

TRIUMF, 4004 Wesbrook Mall, Vancouver, British Columbia, Canada V6T 2A3

Paul W. Percival, Jean-Claude Brodovitch, Siu-Keung Leung and Sunny Sun-Mack

Chemistry Department, Simon Fraser University, Burnaby, British Columbia, Canada V5A 1 S6

Dedicated to Professor Dr. Harry Pfeifer on the occasion of his 65th birthday (Received April 20, 1994)

\section{Surface diffusion / Silica / Cyclohexadienyl radical / Muon spin resonance}

The dynamic behaviour of the cyclohexadienyl radical on a silica surface has been investigated using the avoided level-crossing muon spin resonance technique. Translational motion of the radical on a curved surface leads to partial averaging of the muon-electron hyperfine anisotropy and thus to motional narrowing of the experimental signal. A theoretical model based on a stochastic Liouville formalism allows interpretation of the data in terms of radical diffusion. Arrhenius behaviour with an activation energy of (10.8 \pm

* To whom correspondence should be addressed. 
$0.9) \mathrm{kJ} / \mathrm{mol}$ is observed. Specific deviations between theoretical predictions and experimental results indicate that the potential of this technique is not yet fully exploited and that the model can be further refined.

Das dynamische Verhalten des auf der Oberfläche von Silica adsorbierten Cyclohexadienylradikals wurde untersucht mittels Avoided-Level-Crossing Myon-Spin-Resonanz. Die Translation des Radikals auf der gekrümmten Oberfläche führt zu einer Teilausmittelung der Anisotropie der Myon-Elektron-Hyperfeinwechselwirkung. Sie beeinflußt damit die Linienbreite der untersuchten Resonanz. Diese wurde interpretiert auf der Basis der stochastischen Liouville-Gleichung für die Diffusion des Radikals, wobei Arrheniusverhalten mit einer Aktivierungsenergie von $(10.8 \pm 0.9) \mathrm{kJ} / \mathrm{mol}$ gefunden wurde. Bestimmte Abweichungen zwischen theoretischen Voraussagen und experimentellen Resultaten zeigen, daß das Potential der Technik noch nicht vollständig ausgeschöpft ist und daß das Modell noch verfeinert werden kann.

\section{Introduction}

The motion of molecules both on and off the surface is of fundamental interest in heterogenous catalysis since it determines the turnover frequency and the balance between unimolecular and bimolecular reactions. Nuclear magnetic resonance is well suited to study the mobility of adsorbed diamagnetic molecules, and pioneering work concerned with the surface diffusion of benzene on silica gel was conducted in Leipzig [1-4] and in Hannover [5]. It was shown that intramolecular and intermolecular dipolar protonproton interactions between adsorbate molecules, and furthermore the interaction with surface hydroxyl groups and with paramagnetic impurities in the adsorbent, are important for the relaxation of the protons. Relaxation times were found to lie in the range of $1-1000 \mathrm{~ms}$ depending on surface coverage and on the degree of deuteration of adsorbate and surface [3, 4]. Further studies of the surface mobility of benzene were undertaken for an $\mathrm{Al}_{2} \mathrm{O}_{3}[6,7]$ and a graphite [8] surface.

Free radicals ate potential transient intermediates of heterogeneously catalysed processes. Their surface dynamics is just as essential to the performance of a catalytic process as is the dynamics of diamagnetic reactants and products. Due to the open shell electronic character and the modified structure and energetics of the molecular orbitals, the interaction of the radical with the surface can be expected to differ considerably from that of the parent diamagnetic molecule from which it was derived. On the other hand, the radical and its parent should behave similarly if the surface interaction is dominated by van der Waals forces.

Radicals are less easily amenable to studies of their surface dynamics than are diamagnetic molecules, since as soon as they are mobile they undergo termination reactions, so that at catalytically relevant temperatures their concentration is usually below the detection limit of most conventional experimental techniques. Recently, a new technique which uses positive muons substituted as polarised spin labels in organic radicals has been de- 
veloped [9]. The muons, obtained from a beam line of a suitable accelerator, are stopped in the sample and incorporated in the radical by addition of muonium atoms $\left(\mathrm{Mu} \equiv \mu^{+} e^{-}\right.$is chemically a light isotope of hydrogen with one-ninth the mass of $\mathrm{H}$ ) across unsaturated bonds. The time evolution of muon spin polarisation is the analogue of a free induction decay in pulsed magnetic resonance [10]. It carries information about the magnetic interaction of the muon with the electron and with nuclear spins. Details of the experimental technique have been given elsewhere [9, 11]. The extreme sensitivity allows the detection of radicals at concentrations down to a single species in the entire sample. It therefore permits the investigation of radicals adsorbed on surfaces under conditions where they show translational mobility. The dynamics is deduced from the partial averaging of the muon-electron hyperfine anisotropy. This was demonstrated for the $\mathrm{Mu}$ adduct to 2,3-dimethyl-1,3-butadiene, for which reorientational correlation times were obtained in the motional narrowing regime [12].

Observation using the transverse field technique is limited by the requirements that radicals are formed within $10^{-9} \mathrm{~s}$, in order to avoid loss of phase coherence of the muon spin, and by the restriction that there is no more than one muon present in the sample at any time. An alternative technique, avoided level-crossing muon spin resonance (ALC- $\mu \mathrm{SR}$ ) in longitudinal magnetic fields, allows the detection of radicals even when they are formed over periods of the order of a microsecond, provided that the transition rate is high enough to produce a siginificant ALC signal during the muon lifetime [13]. It is a time-integral technique which can use the full muon flux of the accelerator since there is no constraint on the number of muons within the sample at any given time. Even under these conditions the concentration of radicals is extremely low, and bimolecular termination reactions are negligible. It was therefore possible using this technique to observe cyclohexadienyl and ethyl radicals adsorbed on silica [14-16].

Over most of the region of a strong external magnetic field, eigenstates of a 3-spin system $\left(\mu^{+}, e^{-}\right.$, and nucleus $p^{+}$are all spin- $-1 / 2$ particles) are pure Zeeman states. Muons injected with their spins parallel or antiparallel to this field are thus in an eigenstate. There is no time evolution and hence no muon depolarisation. The behaviour is different in the region of an avoided crossing, where the eigenstates are mixtures of two Zeeman states. This leads to an oscillation between the two states, and if they differ in the $z$-component of the muon spin, to a decrease in polarisation. It is detected as a resonance in a plot of the muon asymmetry against field strength, which is given by the normalised difference of the positron counts in two sets of detectors placed in the forward and backward directions relative to the initial muon spin [13].

There are different ALC transitions classified according to the change in the total spin quantum number $M$ of the participating Zeeman states [14]. 
The resonance obeying the selection rule $\Delta M=0$, essentially a muonproton spin flip-flop, occurs under isotropic conditions to the first order at a resonance field

$$
B_{r}=\frac{1}{2}\left[\frac{A_{\mu}-A_{\mathrm{p}}}{\gamma_{\mu}-\gamma_{p}}-\frac{A_{\mu}+A_{p}}{\gamma_{e}}\right],
$$

where $A_{\mu}, A_{p}$ are the isotropic hyperfine coupling constants of the muon and the nucleus, and $\gamma_{\mu}, \gamma_{p}$ their gyromagnetic ratios. This transition is driven by the isotropic as well as the anisotropic interaction. In isotropic systems such as liquids, only this type of resonance is observed. Increasing anisotropy of the system broadens the signal until it reaches a static line shape which is, in general, asymmetric for a powder, having equal probability for all radical orientations.

A second type of transition obeys the selection rule $|\Delta M|=1$. It corresponds to a muon spin flip and occurs around

$$
B_{r}=\frac{1}{2}\left[\frac{A_{\mu}}{\gamma_{\mu}}-\frac{A_{\mu}}{\gamma_{e}}\right] .
$$

It is driven by the anisotropic coupling elements of the Hamiltonian [17], and therefore vanishes when the system reorients on a time scale faster than the inverse hyperfine anisotropy. The resonance is thus absent under isotropic conditions but very prominent in solids [18], and it is expected to be a sensitive indicator of small anisotropies.

A strong transition obeying the selection rule $\Delta M=0$ was detected for muonated cyclohexadienyl and methoxycyclohexadienyl radicals adsorbed on quartz powders [15]. It was observed that the resonance broadens with decreasing temperature, and this was ascribed to slower reorientation of the radical. A reorientational correlation time was estimated on the basis of a jump model.

Since then, the theoretical model has been greatly improved. This work provides the first example where the analysis of experimental data is based on a stochastic Liouville formalism which was adapted by Kreitzman [19] to describe the dynamics at avoided level crossings. It treats the effects of translational diffusion on a spherical surface, electron spin exchange and chemical reaction, and it takes into account interaction between resonances. The system under investigation is the muonated cyclohexadienyl radical, $\mathrm{C}_{6} \mathrm{H}_{6} \mathrm{Mu}$, adsorbed on the surface of silica gel. It differs from the diamagnetic benzene molecule only by the presence of the $\mathrm{Mu}$ atom and should therefore have closely the same van der Waals interaction. Since the adsorbent is also a silica gel, as in the early work on benzene by the Leipzig group, this should allow a comparison between the surface mobility of a diamagnetic molecule and a close to related radical. In the latter, the dominant magnetic moment is that of the unpaired electron, which is a factor of 
650 stronger than that of the proton. This has the consequence that the nuclear relaxation rates increase by a large factor. All proton-proton dipolar interactions are now negligible, instead, the dipolar part of the electron nuclear hyperfine coupling is the relevant interaction that causes relaxation. Depending on the distance of the proton from the radical centre, the hyperfine anisotropy may amount to as much as $30 \mathrm{MHz}$ [20]. The experimental time scale of dynamic processes which partly average out the dipolar interactions are thus typically a few tens of nanoseconds, which is the same order of magnitude as correlation times amenable to the study of diamagnetic molecules by means of NMR [3].

For benzene adsorbed on silica gel it was proposed by Michel [3] that the adsorbed molecules undergo fast rotation about the sixfold axis down to temperatures of at least $130 \mathrm{~K}$. This was necessary to explain the temperature dependence of the intramolecular part of the proton $T_{1}$ relaxation data. It was supported further by ${ }^{2} \mathrm{H}$ NMR results of benzene- $d_{6}$ adsorbed on graphite [8] and on alumina [6], which demonstrated the dynamic behaviour and the axial symmetry down to low temperature. The cyclohexadienyl radical is also flat and rigid, and it is plausible that in a monolayer of benzene on silica its behaviour is the same as that of benzene. Its energy is lowest when it uses the maximum possible contact area. It is therefore assumed to lie flat on the surface and to rotate rapidly about the axis perpendicular to the molecular plane, leading to an anisotropic system of axial symmetry. Additional motion by translation around the grains or inside the pores leads to an averaging of the axial anisotropy and renders the system isotropic.

\section{Experimental}

A stainless steel sample cell with a window of $25 \mu \mathrm{m}$ thickness was filled with $3 \mathrm{~g}$ of silica gel (Aldrich Chemical Company, Inc.) which had a particle size of 5-25 $\mu \mathrm{m}$, a BET surface area of $500 \mathrm{~m}^{2} / \mathrm{g}$, a pore volume of $0.75 \mathrm{~cm}^{3} / \mathrm{g}$, an average pore diameter of $6.0 \mathrm{~nm}$, and ferromagnetic impurities of $10 \mathrm{ppm}$. It was heated at $383 \mathrm{~K}$ for 24 hours under vacuum until a pressure of less than $10^{-5}$ mbar was reached. This treatment removes physisorbed water and leaves behind a fully hydroxylated surface.

Benzene was degassed with three freeze-pump-thaw cycles, then adsorbed onto the silica gel to give a nominal monolayer, assuming a molecular cross section of $0.40 \mathrm{~nm}^{2}$ [21]. The isosteric heat of adsorption of benzene on a hydroxylated silica surface amounts to ca. $43 \mathrm{~kJ} / \mathrm{mol}$ [22]. The heat of vaporisation of liquid benzene at room temperature is only $34 \mathrm{~kJ} / \mathrm{mol}$. This makes sure that $\mathrm{C}_{6} \mathrm{H}_{6}$ condenses and spreads on the surface and that it does not form droplets or escape into the gas phase to a significant extent at room temperature.

The ALC experiments were performed using a surface muon beam at TRIUMF in Vancouver. The experimental setup and techniques have been described elsewhere [23]. The applied magnetic field was modulated nearly rectangularly by nominally $\pm 9.75 \mathrm{mT}$, and the asymmetry difference between the up and the down phase is displayed in the 


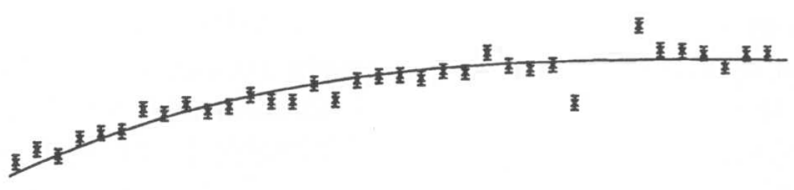

重

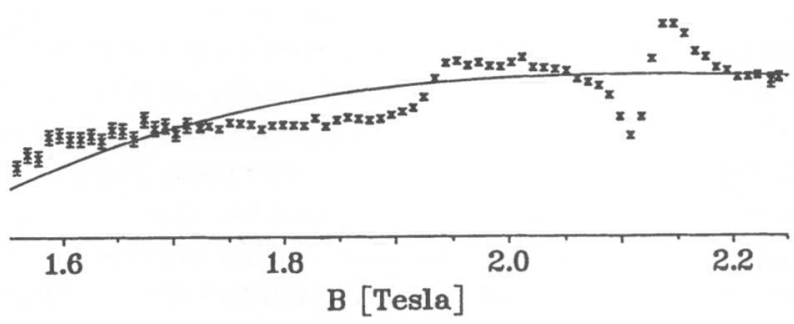

Fig. 1. Raw ALC- $\mu$ SR spectrum of differential asymmetry (arbitrary units) against longitudinal magnetic field, taken at $295 \mathrm{~K}$ (upper), with the fitted background, and at $193 \mathrm{~K}$ (lower) with the same background.

figures to enhance the visibility of the signals by removing the sloping baseline. For the analysis, the theoretical fit function was convoluted with the response function of the magnetic field to account for the exact experimental conditions.

\section{Results and discussion}

For the cyclohexadienyl radical we expect to find four ALC resonances corresponding to the selection rule $\Delta M=0$, which involve the methylene proton (at around $2.1 \mathrm{~T}$ ) and the ortho, meta, and para protons (between $2.7 \mathrm{~T}$ and $3.0 \mathrm{~T}$ ), and the $|\Delta M|=1$ resonance around $1.9 \mathrm{~T}$. Figure 1 displays the raw spectrum obtained in the range of 1.55-2.25 $\mathrm{T}$ for two temperatures. A sharp resonance is clearly observed at $2.1 \mathrm{~T}$. Two further lines were seen at $2.904 \mathrm{~T}$ and at $2.964 \mathrm{~T}$ (ortho and para protons). They clearly identify the radical, but they were too weak for quantitative work and are therefore not used. At $295 \mathrm{~K}$ there seems to be a smooth background (solid line) with no indication of a $|\Delta M|=1$ line. At $193 \mathrm{~K}$, however, a clear but broad feature is observed around $1.9 \mathrm{~T}$. In order to follow the spectral changes with temperature the identical experimental background as indicated in Figure 1 was subtracted in all subsequently displayed spectra. 

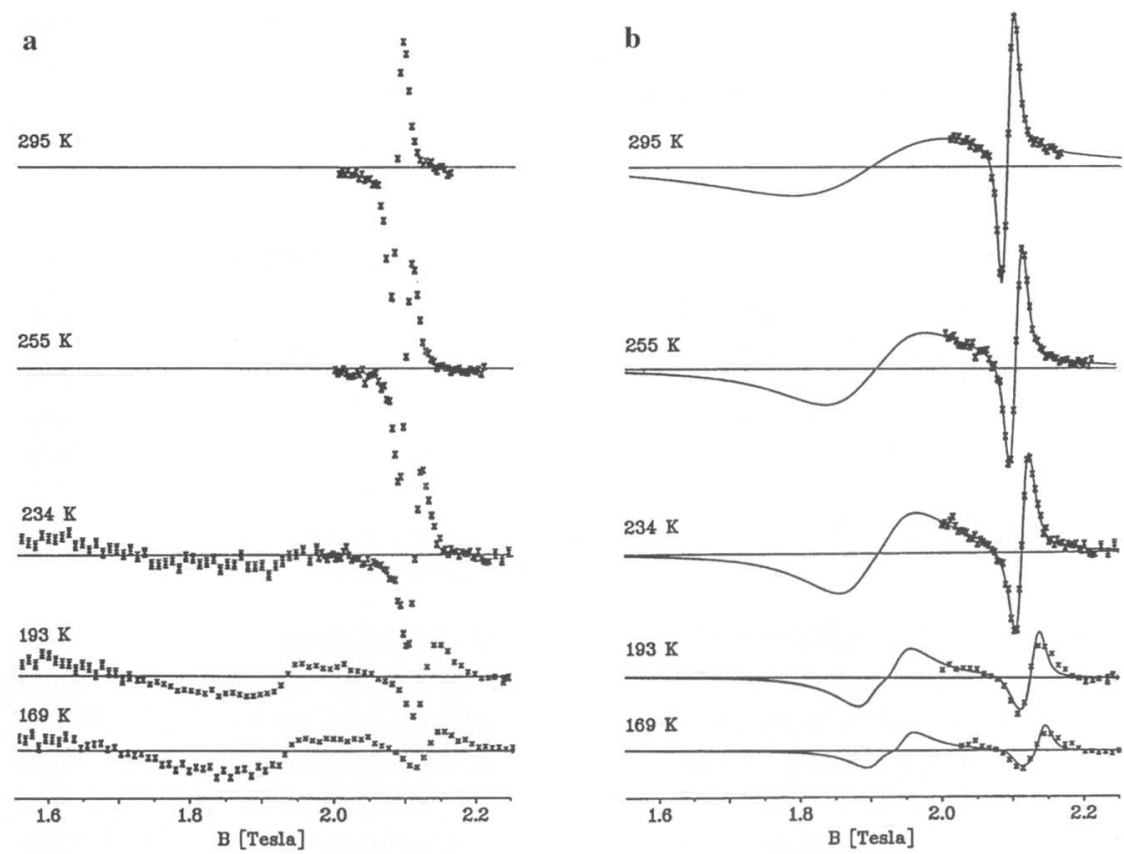

Fig. 2. a) ALC- $\mu$ SR spectra obtained with a nominal monolayer of benzene adsorbed on silica gel at various temperatures. The differential asymmetry, with the background of Fig. 1 subtracted, is displayed. The prominent resonance atound 2.1 Tesla is the $\Delta M=$ 0 muon-proton spin flip-flop transition of the $\mathrm{Mu}$, and the $\mathrm{H}$ bound in the methylene position of the cyclohexadienyl radical. The weak feature around 1.94 Tesla is the $|\Delta M|=1$ muon spin flip resonance. b) Fit of the $\Delta M=0$ resonance with a model considering small angle rotational diffusion, and simulation of the lower field region with the derived fit parameters.

Figure 2a shows a selection of typical spectra taken at different temperatures in the range of $132-295 \mathrm{~K}$. Around 2.1 Tesla the $\Delta M=0$ transition, due to the methylene proton coupling, is observed clearly down to $169 \mathrm{~K}$. With decreasing temperature a broadening together with loss of intensity occurs, and at $132 \mathrm{~K}$ this signal is no longer observable. At $234 \mathrm{~K}$ a weak $|\Delta M|=1$ transition appears, becoming stronger at lower temperature, with a maximum relative intensity around $193 \mathrm{~K}$. Its position, which is directly proportional to the muon spin coupling, Eqn (2), shifts to higher fields with decreasing temperature.

The region of the $\Delta M=0$ transition was analysed in terms of the theoretical model of small angle rotational diffusion of the cyclohexadienyl radical. The fitting function is available in numerical form only. The parameters are the isotropic hyperfine couplings, $A_{\mu}$ and $A_{p}$, their axial anisotropies, $D_{\perp}^{\mu}$ and $D_{\perp}^{p}$, the electron relaxation rate, the coefficient $D_{\text {rot }}$ (which 
Table 1. Results of least squares analysis for the isotropic hyperfine coupling constants of the muon $\left(A_{\mu}\right)$, the methylene proton $\left(A_{p}\right)$, the rotational diffusion coefficient $D_{\text {rot }}$, the experimental scale factor $S$, and the background given by $a+b \cdot\left(B-B_{r}\right)$.

\begin{tabular}{|c|c|c|c|c|c|c|}
\hline $\begin{array}{l}T \\
{[\mathrm{~K}]}\end{array}$ & $\begin{array}{l}A_{\mu}{ }^{\mathrm{a}} \\
{[\mathrm{MHz}]}\end{array}$ & $\begin{array}{l}A_{p} \\
{[\mathrm{MHz}]}\end{array}$ & $\begin{array}{l}D_{\text {rot }} \\
{[\mathrm{MHz}]}\end{array}$ & $S$ & $\begin{array}{l}a \\
{\left[10^{-4}\right]}\end{array}$ & $\begin{array}{l}b \\
{\left[T^{-1}\right]}\end{array}$ \\
\hline 295 & 516.43 & 126.41 & $13.9(4)$ & $0.133(1)$ & $-0.6(4)$ & $0.014(1)$ \\
\hline 275 & 517.93 & 126.48 & $9.9(3)$ & $0.110(1)$ & $2.8(5)$ & $0.019(1)$ \\
\hline 255 & 519.34 & 126.49 & $6.3(2)$ & $0.088(1)$ & $8.3(4)$ & $0.0138(7)$ \\
\hline 234 & 520.91 & 126.52 & $3.8(1)$ & $0.0691(8)$ & $13.2(4)$ & $0.0111(5)$ \\
\hline 213 & 522.48 & 126.77 & $2.7(1)$ & $0.0518(8)$ & $17.8(4)$ & $0.0094(4)$ \\
\hline 193 & 524.05 & 126.96 & $1.6(1)$ & $0.0309(8)$ & $22.2(4)$ & $0.0103(5)$ \\
\hline 169 & 525.85 & 127.83 & $1.1(2)$ & $0.0158(7)$ & $26.2(3)$ & $0.0031(4)$ \\
\hline
\end{tabular}

a Parameter fixed in the fit.

was called $v_{r}$ in [19]) for isotropic rotational diffusion by translation of the radical on the outer or inner surface of a sphere of radius $R$, the signal amplitude $S$, which is proportional to the radical yield, and a sloping background. Of these, only $A_{p}, D_{\text {rot }}, S$, and the background parameters were treated as variables. They are listed in Table 1 . The anisotropic hyperfine parameters were taken as constant. An electron relaxation rate of $0.25 \mathrm{MHz}$ as determined for the liquid by Heming et al. [24] was used at all temperatures, but it has no significant effect on the results. This rate is not strictly temperature independent and has a maximum for $\omega_{e} \tau=1$, which is the case when $\tau$ is of the order of a few picoseconds at the magnetic field used here. It is not expected that our systems are on the fast side of this maximum, so that lower temperatures have a lower relaxation rate, and the room temperature value used here represents the maximum to be expected in our temperature range. Based on the experience of experiments with bulk liquid benzene, chemical reaction was neglected.

Over the entire temperature range, the positions of the $|\Delta M|=0$ resonances are shifted to higher fields by ca. $9.2 \mathrm{mT}$ compared with those in bulk liquid benzene at the same temperature [25], which is in agreement with previous observations [15]. $B_{r}$ depends on the difference $A_{\mu}-A_{p}$ Eqn. (1) and, since the two nuclei participating in the resonance are chemically equivalent, it is assumed that both coupling constants contribute proportionally to this shift and that an identical resonance position represents identical values of $A_{\mu}$ and $A_{p}$. On this basis, $A_{\mu}(T)$ was estimated from its value at $295 \mathrm{~K}$ (taken from the liquid at the temperature with identical $B_{r}$ ) and its temperature dependence on the $\mathrm{SiO}_{2}$ surface, $d A / d T$ $=-0.075(6) \mathrm{MHz} / \mathrm{K}$, and used as a non-variable parameter. $A_{\mu}$ is thus slightly higher on a silica surface than in the bulk liquid at the same temperature, as has been noted previously [15]. It is consistent with the above assumption, and in hindsight a justification for it, that the fitted values of 
$A_{p}$ of the methylene proton (Table 1) are also larger for the adsorbed case compared with the liquid [25], and that they exhibit a similar temperature dependence. The error in $A_{\mu}$ is estimated to be $\leq 0.5 \%$, which can affect $D_{\text {rot }}$ by as much as $5 \%$.

$D_{\perp}^{\prime \prime}$ for the muonium-substituted cyclohexadienyl radical was estimated on the basis of the tensor measured for the corresponding $\mathrm{Mu}$ adduct to durene [26]. It was scaled by the ratio of the isotropic coupling constants of the two radicals, and then the dynamic average was taken for fast rotation about the axis perpendicular to the molecular plane, leading to $D_{\perp}^{\mu}=$ $3.18 \mathrm{MHz}$. The corresponding proton anisotropy, $D_{\perp}^{p}=1.00 \mathrm{MHz}$, was estimated by multiplication of the muon hyperfine value with the ratio of magnetic moments, $\gamma_{p} / \gamma_{\mu}=0.3141$. These parameters influence $D_{\text {rot }}$ approximately according to $\left(D_{\perp}^{\mu}-D_{\perp}^{p}\right)^{2}$, so that an uncertainty of $0.1 \mathrm{MHz}$ in $D_{\perp}^{\mu}$-affects the diffusion coefficient by $6 \%$.

The result of the fit over the $\Delta M=0$ resonance and its extrapolation to lower fields is shown in Fig. $2 \mathrm{~b}$. The model works well for the $\Delta M=0$ transition at higher temperatures, but below $240 \mathrm{~K}$ it deviates significantly. Whereas the experimental $\Delta M=0$ line is of Lorentzian shape at all temperatures, the shape of the fit function becomes increasingly asymmetric.

The theoretical prediction deviates from the experimental spectrum also in the region of the $|\Delta M|=1$ resonance, and in Figure $2 \mathrm{~b}$ the discrepancy becomes most severe for the $255 \mathrm{~K}$ spectrum. While a pronounced feature is predicted, only a weak signal is seen in the experimental data. At lower temperatures, the resonance is indeed present in the experiment but always broader than predicted. It is generally not possible to obtain a consistent fit over the entire field range. In the fit over the restricted range the mismatch is accommodated in the temperature dependence of the background (coefficients $a$ and $b$ in Table 1). Since the resonance is the most prominent one in solid benzene $[18,15]$, its near absence for surface adsorbed benzene is judged to be very significant and obviously of dynamic origin. The exact physical process which causes the line to be so weak is unclear at present. Quantitative analysis therefore had to rely on the $\Delta M=0$ line alone.

The orientational dynamics of the hyperfine tensor are reflected in the rotational diffusion coefficients. For the surface adsorbed radical the averaging over all orientations depends on the grain and pore size as well as on its shape, whereby smaller diameters lead to faster rotational diffusion. An Arrhenius plot of the rotational diffusion coefficients is shown in Figure 3. The slightly curved plot is approximately represented by the equation

$$
D_{\text {rot }}=\left(1.1 \pm{ }_{0.4}^{0.6}\right) \times 10^{9} \mathrm{~s}^{-1} \cdot \exp (-1300 \pm 100) K / T \text {. }
$$

The activation energy of $(10.8 \pm 0.9) \mathrm{kJ} / \mathrm{mol}$ is similar to values for the difiusion of the benzene molecule on hydroxylated porous $\mathrm{Al}_{2} \mathrm{O}_{3}(11.5 \mathrm{~kJ} /$ mol [7]) and on silica gel HR (10.5 kJ/mol [4]). Although both the substrate and the mixture of the surfaces are different, the coincidence of the acti- 


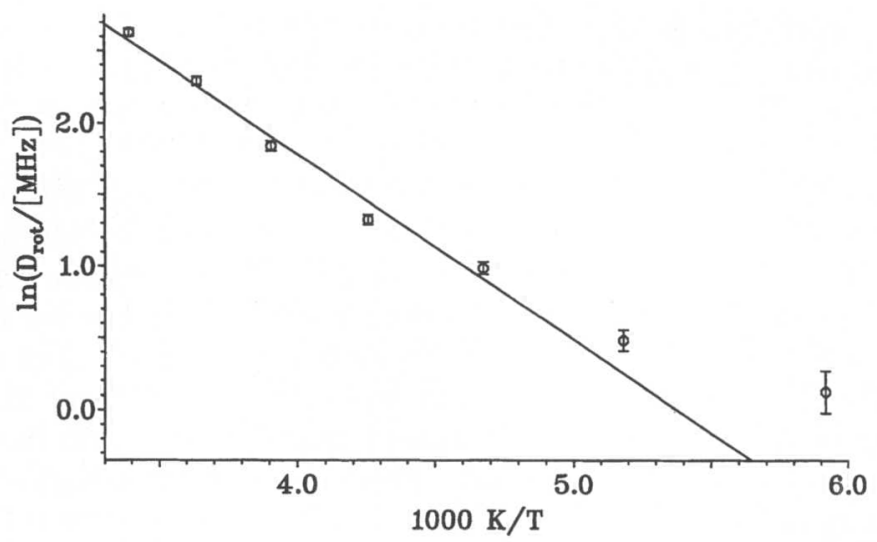

Fig. 3. Arrhenius plot of the rotational diffusion coefficient of the cyclohexadienyl radical, arising from translational diffusion on a spherical surface.

vation energies is striking. Obviously, the radical and its parent molecule have the same activation energy for diffusion on a hydroxylated surface, and, quite unexpectedly, it does not seem to be of primary importance whether this is on a surface of $\mathrm{Al}_{2} \mathrm{O}_{3}$ or of silica. If this coincidence is not just by chance, then it would imply that the binding of the radical to the surface is dominated by van der Waals forces and that the open shell electronic structure plays a minor role. It would also mean that the interaction is mainly with the surface hydroxyl groups which mask the different nature of the underlying substrates. A final interpretation can certainly not be drawn at this point, especially since the extent of surface hydroxylation depends critically on the conditions of adsorbent pretreatment [1]. The early NMR work used up to $300 \mathrm{~K}$ higher temperatures in heating out the silica gel before adsorption of the benzene [2-5]. This point will be further addressed in forthcoming work [27].

Small angle rotational diffusion on the surface of a sphere with radius $R$ is equivalent to two-dimensional translational diffusion on the same surface which for small angles is approximately planar. The two diffusion equations can be interconverted using the substitution $\Phi=r / R$, where $\Phi$ is the rotation angle and $r$ the magnitude of the displacement vector on the surface. This leads to $D_{\text {trans }}=R^{2} D_{\text {rot }}$ [28]. It gives a prefactor of $D_{\text {trans }}^{0}=$ $1 \times 10^{-8} \mathrm{~m}^{2} / \mathrm{s}$ for translational surface diffusion. Due to the irregular shape of the pores [29], and since the molecule could in part also roll instead of just slide on the surface, this is considered to be only a rough estimate. It is expected to be affected by features of the transition state of diffusion, e. g. by radical-surface vibrational modes, and by the average jump distance of the adsorbate which depends on the interaction both with the surface 
and with neighbouring molecules [30]. At $298 \mathrm{~K}$ we obtain $D_{\text {trans }}=1.3$ $\times 10^{-10} \mathrm{~m}^{2} / \mathrm{s}$. The corresponding value in three dimensions amounts to $D_{\text {trans }}=2.0 \times 10^{-10} \mathrm{~m}^{2} / \mathrm{s}$, which is only a factor of 11 below the value for diffusion of benzene in the bulk liquid at the same temperature [31]. The Arrhenius prefactor will be further discussed in upcoming work [27].

The number of sites within reach for the diffusing adsorbed species depends on its root-mean-square displacement on the surface. This is in general also temperature dependent and can cause a prefactor which increases with temperature. The curvature in the Arrhenius plot (Fig. 3) may have to be ascribed to this effect. A further possible origin is the neglect of the inhomogeneity of the surface: Different surface sites lead to a distribution of isotropic muon and proton couplings. This causes an inhomogeneous line broadening which is also averaged out as the species becomes more mobile. The effect would explain why under near-static conditions at low temperatures the theoretical line cannot account for the full experimental width.

It is evident from Table 1 that the signal amplitude also shows a clear trend with temperature. Under the conditions of our experiments $S(T)$ is proportional to the radical yield. Interestingly, it shows a good Arrhenius behaviour, and its activation energy of $6.8 \mathrm{~kJ} / \mathrm{mol}$ is within error the same as that of the $\mathrm{Mu}$ addition reaction to benzene in the gas phase [32]. It is unlikely that this is a pure coincidence, rather it could be taken as evidence that the activated reaction of radical formation competes for Mu with another, unspecified reaction of first or pseudo-first order kinetics, so that the radical is simply not formed at low temperatures. In agreement with this interpretation is the fact that radicals formed on silica by $\mathrm{Mu}$ addition to dienes, which is much less activated [32], are observed down to far lower temperatures.

Further measurements with benzene on non-porous spherical silica grains of narrow size distribution are being written up [27]. They include measurements of the coverage dependence, and exploit also platinum and palladium loaded material, i. e. real catalysts. The systems are more accurate representations of the present theoretical model and are thus expected to allow a more detailed interpretation.

\section{Acknowledgments}

Financial support by the Swiss National Science Foundation and the Natural Sciences and Engineering Research Council of Canada is gratefully acknowledged. TRIUMF is funded through the National Research Council of Canada.

\section{References}

1. H. Pfeifer, in NMR, Basic Principles and Progress, Vol. 7, P. Diehl, E. Fluck and R. Kosfeld, eds., Springer, Berlin, 1972. 
2. D. Freude, Z. Phys. Chem. (Leipzig) 242 (1969) 57.

3. D. Michel, Z. Naturforsch. 23a (1968) 339.

4. H. Winkler, M. Nagel, D. Michel and H. Pfeifer, Z. Phys. Chem. (Leipzig) 248 (1971) 17.

5. B. Boddenberg, R. Haul and G. Opperman, Naturwissenschaften 56 (1969) 635.

6. B. Boddenberg and B. Beerwerth, J. Phys. Chem. 93 (1989) 1435.

7. B. Boddenberg and B. Beerwerth, J. Phys. Chem. 93 (1989) 1440.

8. B. Boddenberg and R. Grosse, Z. Naturforsch. 43a (1988) 497.

9. E. Roduner, The Positive Muon as a Probe in Free Radical Chemistry. Potential and Limitations of the $\mu S R$ Techniques, Lecture Notes in Chemistry, Vol. 49, Springer, Heidelberg, 1988.

10. E. Roduner, Chem. Soc. Rev. 22 (1993) 337.

11. E. Roduner, M. Schwager and M. Shelley, in Radicals on Surfaces, eds. A. Lund and C. J. Rhodes, series on Molecular Organization and Engineering, Klewer Academic Publishers, in press.

12. M. Heming and E. Roduner, Surf. Sci. 189/190 (1987) 535.

13. M. Heming, E. Roduner, B. D. Patterson, W. Odermatt, J. Schneider, H. Baumeler and H. Keller, Chem. Phys. Lett. 128 (1986) 100.

14. I. D. Reid, T. Azuma and E. Roduner, Nature 345 (1990) 328.

15. I. D. Reid, T. Azuma and E. Roduner, Hyperfine Interact. 65 (1990) 879.

16. M. Schwager, E. Roduner, I. D. Reid, P. W. Percival, J.-C. Brodovitch, S. Wlodek and R. F. Marzke, Hyperfine Interact. 87 (1994) 859.

17. E. Roduner, I. D. Reid, M. Riccó and R. De Renzi, Ber. Bunsenges. Phys. Chem. 93 (1989) 1194.

18. E. Roduner, Hyperfine Interact. 65 (1990) 857.

19. S. Kreitzman and E. Roduner, Chem. Phys. 189 (1994) in press.

20. A. Carrington and A. D. McLachlan, Introduction to Magnetic Resonance, Harper, New York, 1967.

21. S. I. Gregg and K. S. W. Sing, Adsorption, Surface Area and Porosity, Academic, London, 1967.

22. A. V. Kiselev and V. I. Lygin, Infrared Spectra of Surface Compounds, Wiley, New York, 1975.

23. P. W. Percival, J. C. Brodovitch, S. K. Leung, D. Yu, R. F. Kiefl, G. M. Luke, K. Venkateswaran and S. F. J. Cox, Chem. Phys. 127 (1988) 137.

24. M. Heming, E. Roduner, I. D. Reid, P. W. F. Louwrier, J. W. Schneider, H. Keller, W. Odermatt, B. D. Patterson, H. Simmler, B. Pümpin and I. M. Savić, Chem. Phys. 129 (1989) 335.

25. D. Yu, P. W. Percival, J. C. Brodovitch, S. K. Leung, R. F. Kiefl, K. Venkateswaran and S. F. J. Cox, Chem. Phys. 142 (1990) 229.

26. E. Roduner, Chem. Phys. Lett. 81 (1981) 191.

27. M. Schwager, H. Dilger, E. Roduner, I. D. Reid, P. W. Percival and A. Baiker, Chem. Phys. 189 (1994) in press.

28. P. A. Flinn, Diffusion in Solids and Liquids, in: Application of Mössbauer Spectroscopy, R. L. Cohen, ed., Vol. 2, Academic Press, New York, 1980.

29. J. M. Drake and J. Klafter, Phys. Today (1990) 46.

30. K. D. Dobbs and D. J. Doren, J. Chem. Phys. 97 (1992) 3722.

31. A. F. Collings and R. Mills, Trans. Faraday Soc. 66 (1970) 2761.

32. E. Roduner, P. W. F. Louwrier, G. A. Brinkman, D. M. Garner, I. D. Reid, D. Arseneau, M. Senba, and D. G. Fleming, Ber. Bunsenges. Phys. Chem. 94 (1990) 1211. 